\title{
Photoluminescence of Lead Sulfide Quantum Dots of Different Sizes in a Nanoporous Silicate Glass Matrix
}

Aleksandr P. Litvin

ITMO University, litvin88@gmail.com

Anton Babaev

ITMO University

Peter Parfenov

ITMO University

See next page for additional authors

Follow this and additional works at: https://arrow.tudublin.ie/engscheleart2

Part of the Electrical and Computer Engineering Commons

\section{Recommended Citation}

Litvin, Aleksandr \& Babaev, Anton \& Parfenov, P. \& Ushakova, Elena \& Baranov, Mikhail \& Andreeva, Olga \& Berwick, Kevin \& Fedorov, A. \& Baranov, A.. (2017). Photoluminescence of Lead Sulfide Quantum Dots of Different Sizes in a Nanoporous Silicate Glass Matrix. The Journal of Physical Chemistry C. 121. 10.1021/ acs.jpcc.7b01952.

This Article is brought to you for free and open access by the School of Electrical and Electronic Engineering at ARROW@TU Dublin. It has been accepted for inclusion in Articles by an authorized administrator of ARROW@TU Dublin. For more information, please contact arrow.admin@tudublin.ie, aisling.coyne@tudublin.ie, gerard.connolly@tudublin.ie.

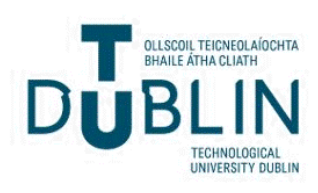


Authors

Aleksandr P. Litvin, Anton Babaev, Peter Parfenov, Elena Ushakova, Mikhail Baranov, Olga Andreeva, Kevin Berwick, Anatoly Fedorov, and Alexander Baranov

This article is available at ARROW@TU Dublin: https://arrow.tudublin.ie/engscheleart2/292 


\title{
Photoluminescence of Lead Sulfide Quantum Dots of Different Sizes in a Nanoporous Silicate Glass Matrix
}

\author{
Aleksandr P. Litvin, ${ }^{* \dagger}{ }^{\dagger}$ Anton A. Babaev, ${ }^{\dagger}$ Peter S. Parfenov, ${ }^{\dagger}$ Elena V. Ushakova, ${ }^{\dagger}$ Mikhail A. Baranov, \\ Olga V. Andreeva, ${ }^{\dagger}$ Kevin Berwick, ${ }^{\dagger}$ Anatoly V. Fedorov, ${ }^{\dagger}$ and Alexander V. Baranov ${ }^{\dagger}$ \\ ${ }^{\dagger}$ ITMO University, Kronverksky pr., 49, St. Petersburg, Russia \\ ${ }^{\ddagger}$ School of Electrical and Electronic Engineering, Dublin Institute of Technology, Kevin Street, Dublin 8, Ireland
}

ABSTRACT: The optical properties of lead sulfide quantum dots (QDs) of different sizes embedded in a nanoporous silicate glass matrix (NSM) are investigated by steady-state and transient photoluminescence spectroscopy. The use of this matrix allows the fabrication of samples with reproducible optical characteristics, for both isolated and close-packed QDs. Low-temperature PL analysis of isolated QDs with sizes of 3.7 and $4.5 \mathrm{~nm}$ shows that the coefficient of temperature shift of the PL position changes sign with reducing QD size because of size-dependent contributions from thermal expansion, mechanical strain, and electron-phonon coupling. The PL intensity is determined by size-dependent splitting of the lowest energy electronic state.

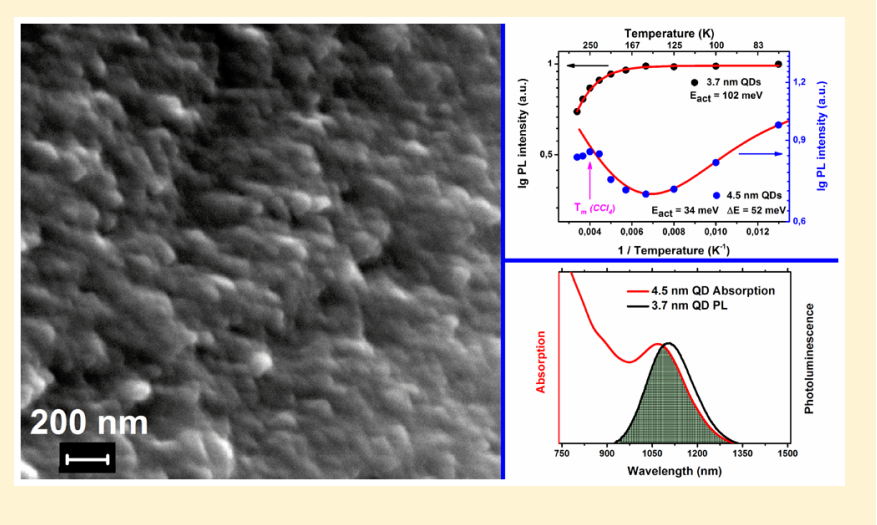

\section{INTRODUCTION}

Colloidal quantum dots (QDs) possess a unique ability to control their optical properties by varying their chemical composition, shape, size, and chemical organic ligands. ${ }^{1}$ Exploiting this ability should allow a wide range of useful devices to be engineered using these materials. In particular, lead sulfide $(\mathrm{PbS})$ QDs with optical transitions in the nearinfrared region are now used in third-generation photovoltaic cells, $^{2-4}$ photodetectors, ${ }^{5-9}$ and light-emitting diodes. ${ }^{10-12}$ However, fundamental research focused on their physical properties remains necessary in order to fully understand the mechanisms governing the relaxation dynamics of QD excitons at the nanoscale.

Identification of a suitable matrix to host these QDs remains an open research question and is crucial for the further development of QD-based devices. ${ }^{13-18}$ Any matrix must provide a homogeneous distribution and controllable density of QDs at a relatively high level, optical transparency, and stability of physicochemical properties. Nanoporous silicate glass matrices (NSM) are very promising materials for QD infiltration because they possess excellent optical transmission over a wide spectral range and high radiation and mechanical resistance. Another important advantage of NSMs is that they allow a controlled variation of the pore diameter, opening the possibility of size-selective infiltration of QDs and study of the optical properties of both isolated and close-packed QDs. Several applications of NSMs filled by various nanoparticles such as fullerene-based optical limiters, ${ }^{19} \mathrm{Ag}$-AgI-based hybrid "plasmon-exciton" nanostructures, ${ }^{20}$ and ammonia vapor sensors $^{21}$ have been reported recently.
In this study, we demonstrate that an NSM can be used as a high quality, stable matrix for studying the optical properties of both isolated and close-packed QDs inside the glass pores. While the close-packed regime allows the study of interactions between QDs such as FRET, the opportunity to study isolated QDs in a host matrix allows the examination of the intrinsic properties of the QDs. This allows us to reveal some unusual photoluminescent properties of $\mathrm{PbS} \mathrm{QDs}$ of different sizes at low temperatures, which are important for a deeper insight into the nature of energy dissipation in lead chalcogenide nanocrystals.

\section{MATERIALS AND EXPERIMENTS}

$\mathrm{PbS}$ QDs with mean diameters of 3.7 and $4.5 \mathrm{~nm}$, capped by $1.8 \mathrm{~nm}$ long oleic acid ligands, have been synthesized by the hot-injection method and dissolved in carbon tetrachloride $\left.\left(\mathrm{CCl}_{4}\right)\right)^{22}$ The QDs are then embedded into a nanoporous silicate matrix (NSM, with $17 \mathrm{~nm}$ pores, made of $\mathrm{Na}_{2} \mathrm{O}-$ $\mathrm{B}_{2} \mathrm{O}_{3}-\mathrm{SiO}_{2}$ glass $^{19}$ ) and a porous paper matrix (PPM, with micrometer-scale pores, 388 grade Sartorius filter paper $^{23}$ ).

The samples of NSM with embedded QDs were prepared as follows. Plates of NSM of $1 \mathrm{~mm}$ thickness were annealed at 530 ${ }^{\circ} \mathrm{C}$ for $2 \mathrm{~h}$ to allow pore purification. Annealing ensures the absence of impurities adsorbed from the air and promotes better penetration of any filling compounds. Next, the NSM plate was placed onto the QD solution/air interface. The concentration of the QDs in the solutions used ranged from

Received: February 28, 2017

Revised: March 24, 2017

Published: March 27, 2017 


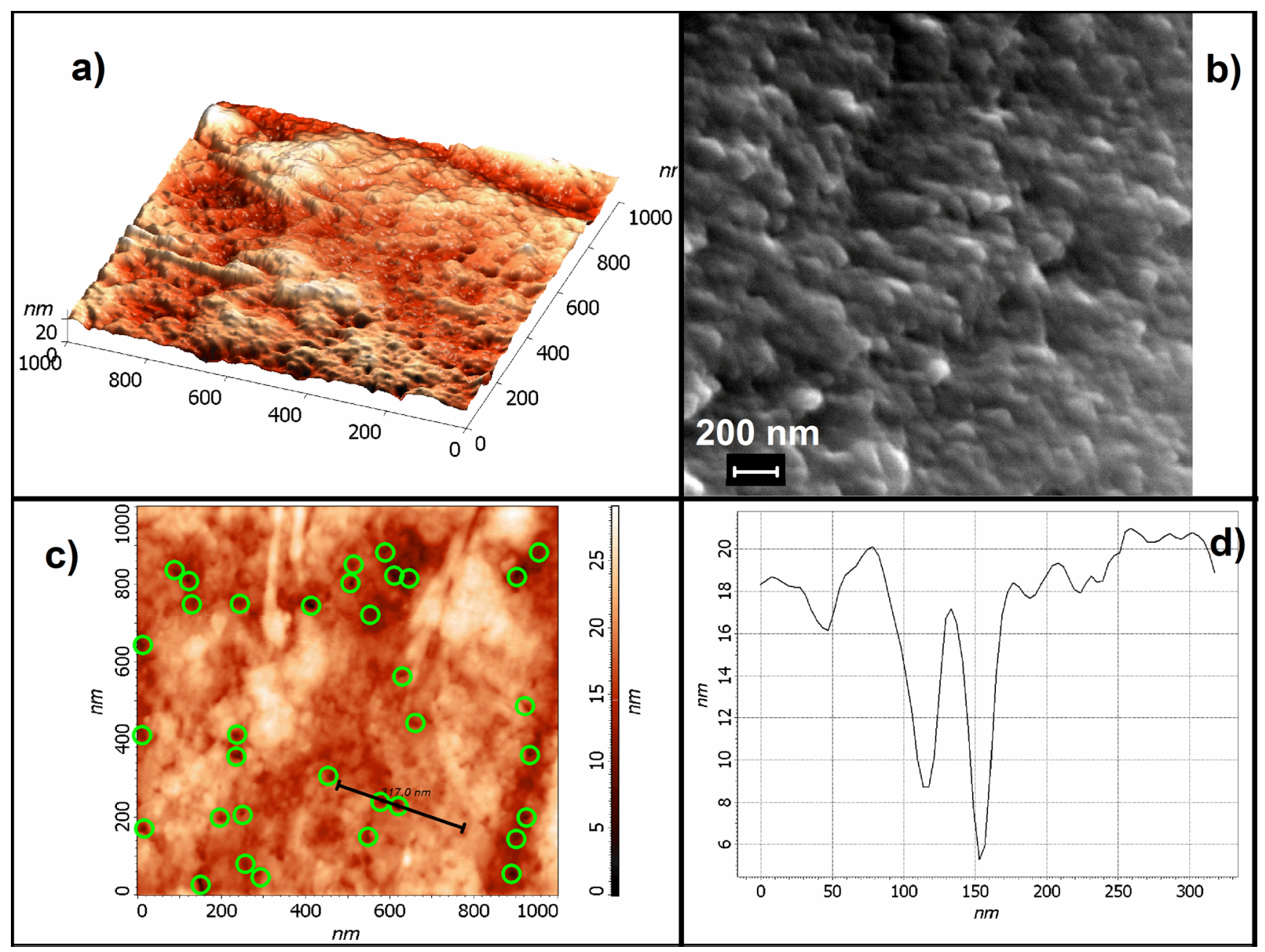

Figure 1. 3D topography of the NSM cross section obtained by AFM (a) and SEM (b). (c) Nanoscale relief of the NSM surface with a few pronounced pores obtained by AFM. (d) The cross section of the NSM surface along the line shown in (c).

$10^{-4}$ to $10^{-5} \mathrm{M}$, in order to provide different $\mathrm{QD}$ concentrations inside the matrix. The solutions were subjected to $30 \mathrm{~min}$ of ultrasonication to prevent $\mathrm{QD}$ aggregation. After several seconds, the color of the plate becomes light-brown, indicating that the QDs have soaked into the plate. Next, the plate was dipped completely into the colloidal solution to allow homogeneous infiltration of the QDs. To finish, the plate was rinsed several times in $\mathrm{CCl}_{4}$ solution in order to ensure that the optical responses collected arise from the QDs located inside the NSM volume.

The infiltration and optical properties of QDs in a PPM have been previously investigated in detail. ${ }^{23}$ So the samples of the QDs in the PPM were used as a reference. PPM is intended for filtration of residuals with typical sizes larger than $10 \mu \mathrm{m}$ and is composed of cellulosic fibers with a diameter of a few micrometers, which effectively adsorb semiconductor QDs on their surface. ${ }^{23}$ With increasing QD concentration, QDs start to form agglomerates at the fiber surface, and formation of closepacked ensembles of interacting QDs affects their optical properties. ${ }^{24}$ Samples of PPM were dipped into $\mathrm{CCl}_{4}$ colloidal solutions with QD concentrations of $\sim 10^{-5} \mathrm{M}$ for $30 \mathrm{~s}$. After that the samples were dried for $1 \mathrm{~h}$ at $50{ }^{\circ} \mathrm{C}$ to evaporate the residual $\mathrm{CCl}_{4}$.

Topographic atomic force microscopy (AFM) images of a porous glass surface were obtained by atomic force microscope
Solver Pro-M (NT-MDT, Russia) with NSG01 probe (10 nm curvature radii) in semicontact mode. Scanning electron microscopy (SEM) was performed using a Merlin-Zeiss microscope at $15 \mathrm{kV}$.

The optical properties of the samples were studied using a Shimadzu-UV3600 spectrophotometer and a purpose-built setup for the PL analysis of nanostructures in the $\mathrm{NIR}^{25} \mathrm{~A}$ $633 \mathrm{~nm}$ laser excitation was used for steady-state PL analysis, and $532 \mathrm{~nm}$ excitation was used for transient measurements. Raw PL spectra were normalized, taking into account the spectral sensitivity of the experimental setup in order to extract the emission spectra. ${ }^{26}$

\section{RESULTS AND DISCUSSION}

Nanoporous Silicate Glass Matrix. The surface of the NSM was examined by atomic force and electron microscopies. Figures $1 \mathrm{a}$ and $1 \mathrm{~b}$ demonstrate the 3D topography of the NSM cleavage obtained by AFM and SEM, respectively. The images show nanoscale relief of the NSM. Since the sizes of the pores are close to the probe tip radius, we can distinguish only a few pores on AFM images, as shown in Figure 1c by green circles. The cross section of the NSM surface topography around two closely located pronounced pores is shown in Figure 1d and indicates the pore diameter of ca. $20 \mathrm{~nm}$. The SEM image 
a)

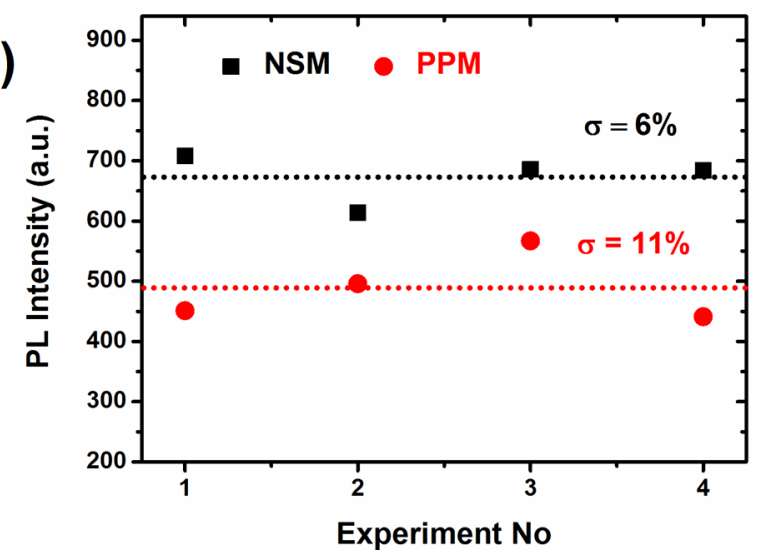

b)

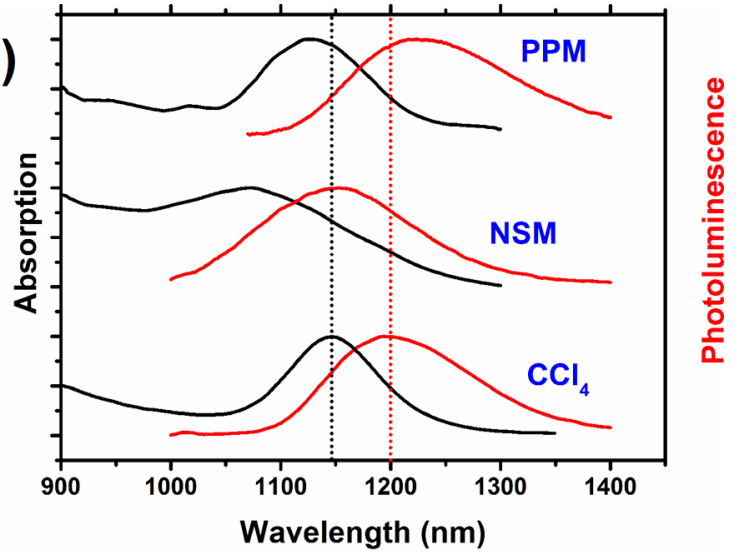

Figure 2. (a) PL peak intensities from four different areas of the porous glass and filter paper with embedded $4.5 \mathrm{~nm}$ QDs. (b) Absorption (black lines) and PL (red lines) spectra of $4.5 \mathrm{~nm} \mathrm{PbS}$ QDs in PPM, NSM, and $\mathrm{CCl}_{4}$. Vertical dashed lines indicate the centers of the absorption and PL bands for QDs in a $\mathrm{CCl}_{4}$ colloidal solution.
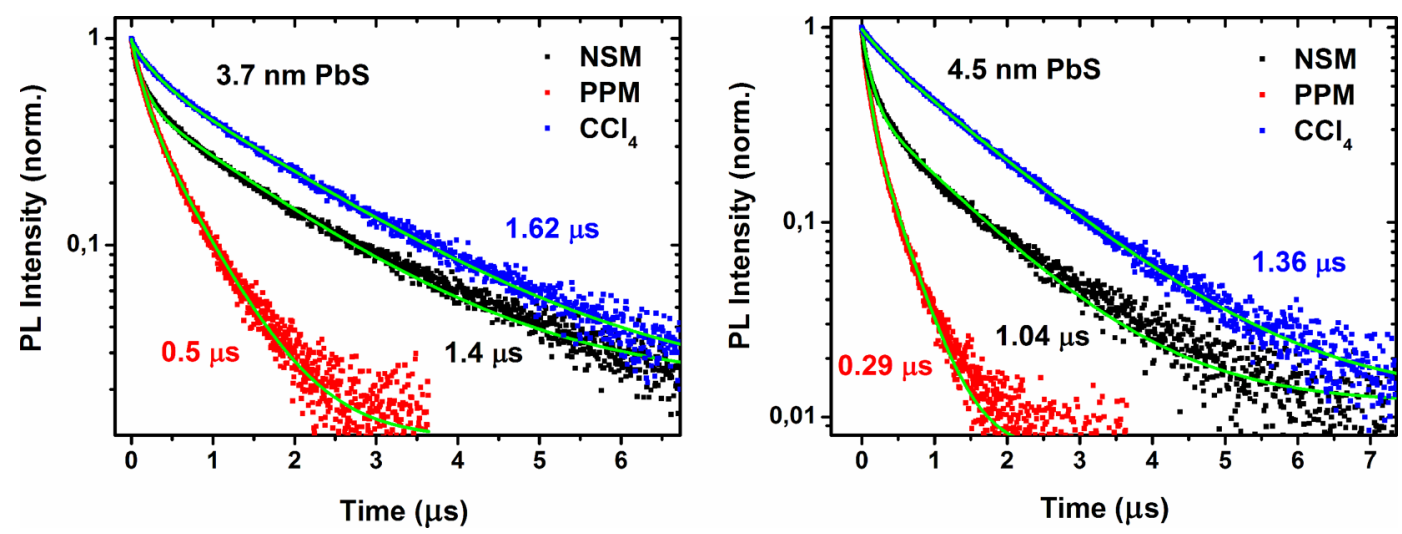

Figure 3. PL decay curves recorded for 3.7 and $4.5 \mathrm{~nm} \mathrm{PbS}$ QDs in colloidal solution (blue), in porous glass (black), and in filter paper (red). Intensity-weighted average PL lifetimes are shown.

(Figure 1b) shows also pronounced structures with transversal sizes of around $20 \mathrm{~nm}$.

Next, we examined the homogeneity of the spatial QD distribution inside the NSM volume. In order to do this, we compared the optical responses from the NSM sample with those from the PPM samples, which are known to possess homogeneous QD distributions. ${ }^{23}$ We chose four different areas on the NSM and PPM samples to record PL spectra. The optical density of the NSM plates at the PL excitation and emission wavelengths was less than 0.1 , allowing the examination of the distribution of the QDs inside the sample volume. Results for samples with $4.5 \mathrm{~nm} \mathrm{PbS}$ QDs are shown in Figure 2a. The black squares and red circles represent the PL peak intensities for the NSM and PPM, respectively. The dotted lines indicate mean values and the standard deviations are listed in the legend. These results demonstrate that we obtained good homogeneity in the QD distribution, in both the NSM and PPM samples. Clearly, the NSM matrix exhibits superior homogeneity in the QD distribution, giving reproducible PL responses.

Noninteracting Regime. Our analysis of the steady-state absorption and PL, as well as the PL decay times, of the NSM filled with QDs shows that a system of noninteracting QDs can be formed inside the NSM. This is supported by Figure $2 b$, where a comparison between the absorption (black lines) and PL (red lines) spectra of $4.5 \mathrm{~nm}$ PbS QDs in PPM, NSM and $\mathrm{CCl}_{4}$ is shown. The samples of QDs in the NSM and the PPM were obtained by using colloidal solutions with QD concentrations of $\sim 10^{-5} \mathrm{M}$. The absorption bands of the QDs in both the NSM and PPM are blue-shifted compared to those from the QD solution. The magnitude of the shift is dependent on the specific matrix and, in particular, their dielectric constant. The PL band of the PPM sample is redshifted, indicating FRET is occurring between the QDs. ${ }^{27}$ So for PPM we are dealing with close-packed interacting QDs with energy transfer occurring between QDs which differ slightly in size. ${ }^{24}$ In contrast, the NSM sample shows no spectral evidence of QD interaction at the QD concentration used, since its PL peak is shifted in the same direction as the absorption peak with an unchanged Stokes shift. It appears that there is a more homogeneous spatial distribution of isolated QDs in the NSM than in the PPM, where conglomerates of close-packed QDs are formed in the pores.

This conclusion is supported by an analysis of the PL decay curves for QDs in $\mathrm{CCl}_{4}, \mathrm{NSM}$, and PPM, performed for 3.7 and $4.5 \mathrm{~nm} \mathrm{PbS}$ QDs and presented in Figure 3. The PL decay curves obtained for the samples are well fitted by a sum of two exponential functions. We calculated intensity-weighted average PL lifetimes, also shown in Figure 3. Very long decay times for $\mathrm{PbS}$ QD colloidal solutions have been previously reported in several papers ${ }^{22,28-31}$ and were explained by the contribution of several electronic states to the $\mathrm{PL}$ signal. $^{22,32}$ To detect interactions between QDs in the different matrices, we performed transient PL analysis with $20 \mathrm{~nm}$ spectral selection 
inside the luminescence bands of $4.5 \mathrm{~nm}$ QDs in $\mathrm{CCl}_{4}, \mathrm{NSM}$, and PPM. The corresponding intensity-weighted average PL lifetimes were calculated for each band and then were normalized by the value, which is the average at the specified spectral range. Spectral dependencies of the normalized intensity-weighted average PL lifetimes are shown in Figure 4. As expected, no notable spectral dependence of PL lifetimes

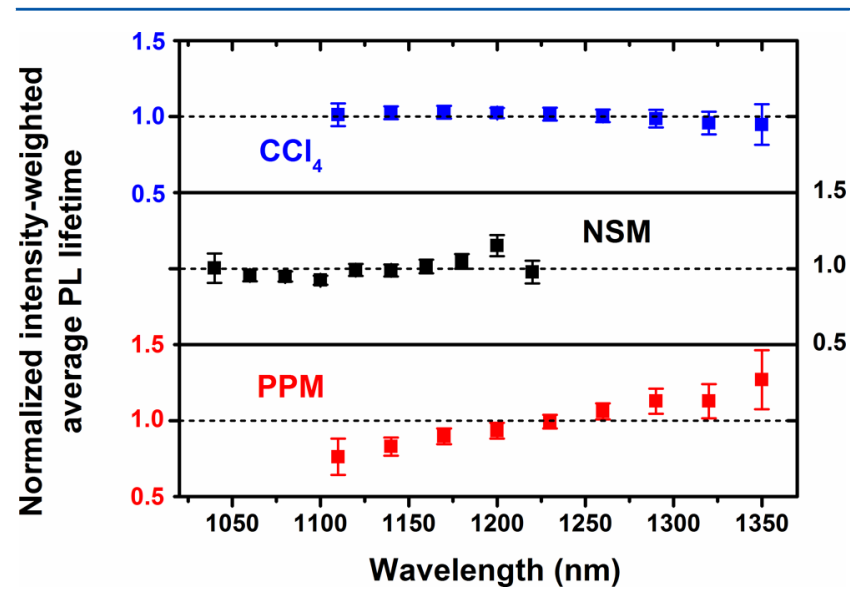

Figure 4. Normalized intensity-weighted average PL lifetimes obtained at different wavelengths for $4.5 \mathrm{~nm} \mathrm{PbS}$ QDs in colloidal solution (blue), porous glass (black), and filter paper (red).

has been observed for the dilute solution of noninteracting QDs. In contrast, the average PL lifetimes of QDs embedded in the PPM reveal a pronounced wavelength dependence, indicating that quenching of smaller QDs inside a quasimonodispersed ensemble due to energy transfer ${ }^{24,27}$ is taking place. This fact, together with the much shorter PL decay times compared to the QDs in the $\mathrm{CCl}_{4}$, indicates the formation of conglomerates, composed of strongly interacting QDs. In contrast to the PPM samples, PbS QDs in the NSM demonstrate PL lifetimes with only a slight wavelength dependence. The calculated PL decay times are much closer to those observed in colloidal QD solutions; any slight differences may be ascribed to the difference in the dielectric constant of the matrix or slight optical quenching by the matrix. ${ }^{33}$ This observation, together with the minor wavelength dependence of PL lifetimes, shows that the dynamics of energy relaxation for QDs embedded in the NSM are typical of those from isolated QDs. In other words, from an analysis of the absorption and steady-state and transient PL responses of the samples, it is clear that the NSM allows the investigation of the optical properties of individual noninteracting QDs.

Low-Temperature PL Analysis. Since the use of a nanoporous silicate glass matrix allows us to make samples with noninteracting QDs and reproducible optical responses, we were able to explore the PL responses from isolated $\mathrm{PbS}$ QDs of different sizes, including the temperature dependencies of PL properties. We performed PL analysis for 3.7 and $4.5 \mathrm{~nm}$ $\mathrm{PbS}$ QDs embedded in an NSM from colloidal solutions with QD concentrations of $10^{-5} \mathrm{M}$ in the temperature range 77$300 \mathrm{~K}$. Figure 5 represents the temperature dependence of the fwhm of the PL band from the $4.5 \mathrm{~nm}$ PbS QDs. The fwhm of the PL band suffers conventional broadening with increasing temperature. The dependence of the fwhm on temperature can be described as follows: ${ }^{34-36}$

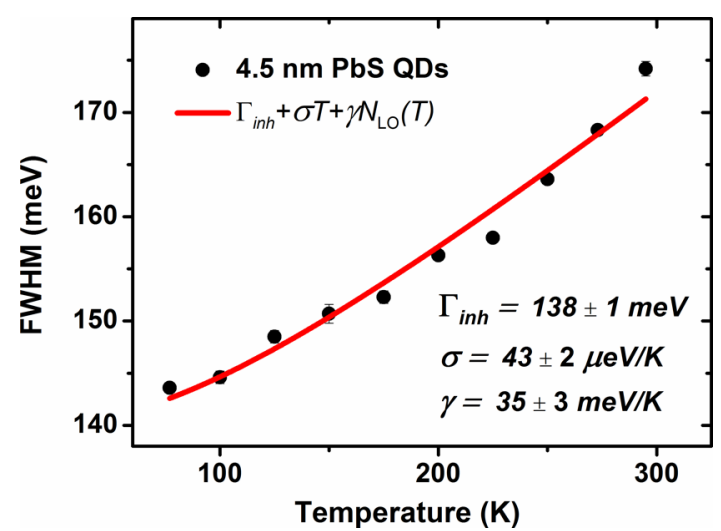

Figure 5. The fwhm temperature dependence for $4.5 \mathrm{~nm} \mathrm{PbS}$ QDs in NSM. The red line is the fit to the experimental data (solid dots) with eq 1 . Fitting parameters are $\sigma=43 \mu \mathrm{eV} / \mathrm{K}, \gamma=35 \mathrm{meV} / \mathrm{K}$, and $\Gamma_{\text {inh }}=$ $138 \mathrm{meV}$, as shown in the legend.

$$
\mathrm{fwhm}(T)=\Gamma_{\text {inh }}+\sigma T+\gamma\left[\exp \left(\frac{E_{\mathrm{LO}}}{k_{\mathrm{B}} T}\right)\right]^{-1}
$$

where $\Gamma_{\text {inh }}$ is the QD inhomogeneous distribution width which is temperature-independent, arising mainly from fluctuations in the size and shape of the QDs in the ensemble studied. The coefficients $\sigma$ and $\gamma$ are the exciton-acoustic phonon and exciton-LO phonon coupling coefficients respectively, $E_{\mathrm{LO}}$ is the LO phonon energy, and $k_{\mathrm{B}}$ is the Boltzmann constant. Fitting the temperature dependence of the fwhm for the $4.5 \mathrm{~nm}$ $\mathrm{PbS}$ QDs in the NSM by eq 1 with fitting parameters $\sigma, \gamma, \Gamma_{\mathrm{inh}}$, and a fixed value of $E_{\mathrm{LO}}=25.4 \mathrm{meV}$ (the bulk value at $296 \mathrm{~K}^{37}$ ) is shown in Figure 5 by the red solid line. Values for $\sigma=43$ $\mu \mathrm{eV} / \mathrm{K}, \gamma=35 \mathrm{meV} / \mathrm{K}$, and $\Gamma_{\text {inh }}=138 \mathrm{meV}$ have been obtained from the fitting procedure. The $\sigma$ and $\gamma$ values are close to those reported previously for $5 \mathrm{~nm}$ QDs in silicate glass ${ }^{34}$ while the $\Gamma_{\text {inh }}$ value is near the width expected at $0 \mathrm{~K}$ from a rough extrapolation of the fwhm temperature dependence below 125 K.

Figure 6 represents a temperature dependence of the PL peak position for 3.7 and $4.5 \mathrm{~nm} \mathrm{PbS}$ QDs embedded in an NSM. The shifts have been approximated by the Varshni equation: ${ }^{38}$

$$
E_{\mathrm{g}}(T)=E_{\mathrm{g} 0}-\alpha \frac{T^{2}}{T+\beta}
$$

where $E_{\mathrm{g} 0}$ is the bandgap width at $0 \mathrm{~K}, \alpha$ is the coefficient of temperature shift of the bandgap energy, and $\beta$ is the temperature-independent parameter which is usually close to the Debye temperature. An approximation of the PL peak position for 3.7 and $4.5 \mathrm{~nm} \mathrm{PbS}$ QDs using the Varshni relation with $\beta=145 \mathrm{~K}$ gives coefficients of $\alpha$ of $-120 \pm 10$ and $180 \pm$ $10 \mu \mathrm{eV} / \mathrm{K}$, respectively, and $E_{\mathrm{g} 0}$ of $1.178 \pm 0.001$ and $1.077 \pm$ $0.001 \mathrm{eV}$, respectively. Olkhovets and co-workers first showed that lead chalcogenide QDs possess a strongly size-dependent temperature coefficient of bandgap energy. ${ }^{38}$ They demonstrated that the temperature coefficient of the lowest excited state in the absorption spectra varies over a wide range, from negative values up to the positive value from the bulk material. This is explained by considering the different contributions from thermal expansion, mechanical strain, and electronphonon coupling, whose size dependencies are especially pronounced for the smallest QDs. 

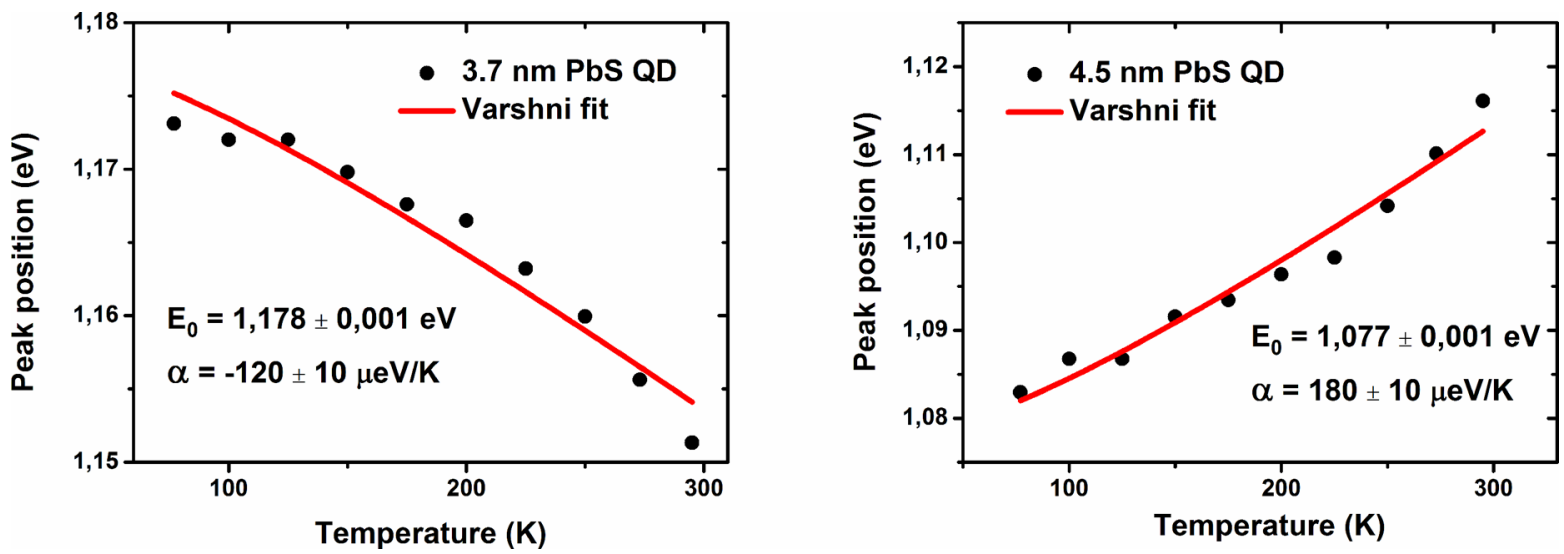

Figure 6. Temperature dependence of the PL peak position for 3.7 and $4.5 \mathrm{~nm}$ PbS QDs embedded in the NSM.

This trend was also confirmed in further experiments for lead chalcogenide absorption ${ }^{39}$ and PL temperature dependencies. ${ }^{40}$ Caram et al. have recently observed different temperature dependencies in the PL position for PbS QDs of different sizes and ascribed this to the redistribution of the state population between two emitting electronic states. ${ }^{32}$ In our case, PbS QDs in the NSM did not demonstrate two distinct PL bands, and any variation in the coefficient $\alpha$ should be considered to be a result of the size dependence of various thermal contributions. For 3-4 nm PbS QDs, thermal expansion of the wave function envelope and intraband deformation-potential coupling of electrons to acoustic phonons make a negative contribution to $\alpha$, leading to a sign change in $\alpha$. $^{38}$

An analysis of the PL intensity is shown in Figure 7, highlighting another distinction between the luminescent

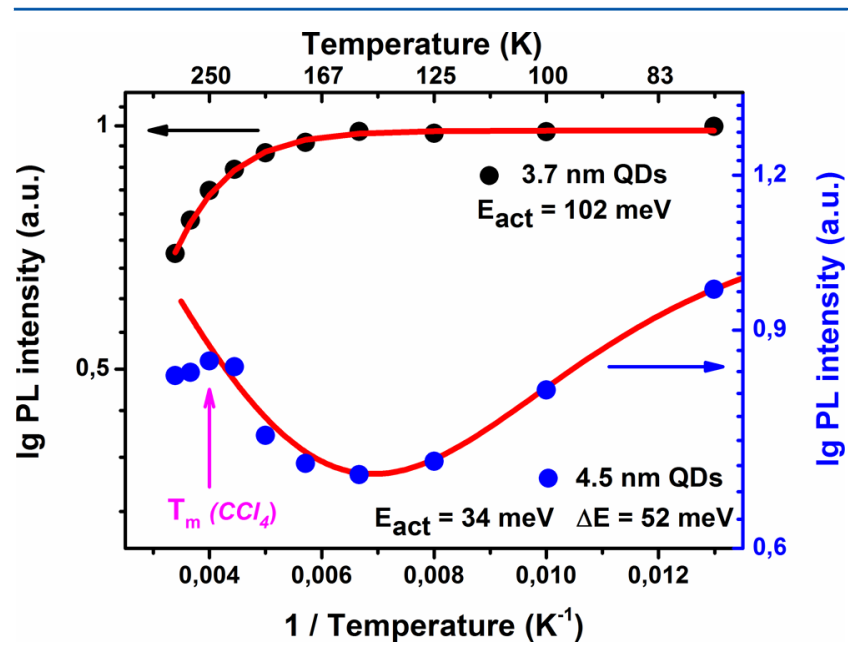

Figure 7. Temperature dependencies of PL intensities for 3.7 and 4.5 $\mathrm{nm} \mathrm{PbS}$ QDs embedded in an NSM.

properties of 3.7 and $4.5 \mathrm{~nm} \mathrm{PbS}$ QDs. The temperature dependence of the PL intensity for $3.7 \mathrm{~nm}$ QDs is shown by black circles. The dependence for temperatures $>60 \mathrm{~K}$ can be well fitted by the standard expression (3) with an activation energy $E_{\mathrm{A}}: 41$

$$
I_{\mathrm{PL}}=\frac{I_{0}}{1+A \exp \left(-\frac{E_{\mathrm{A}}}{k_{\mathrm{B}} T}\right)}
$$

The estimated activation energy of $102 \pm 6 \mathrm{meV}$ was found to be close to that for CdTe, ${ }^{42,43} \mathrm{PbTe}^{44}$ and PbSe/CdSe QDs. ${ }^{45}$ Activation energies of $100-130 \mathrm{meV}$ have been recently obtained for $\mathrm{PbS}$ QDs from theoretical calculations using a model which includes several emitting states. ${ }^{32}$ Zhao et al. have also reported activation energies of $100-130 \mathrm{meV}$ for 3-4 nm $\mathrm{PbS}$ QDs capped by oleic acid in PMMA. ${ }^{40}$ A relatively slight reduction in the PL intensity with increasing temperature has been previously noted by Turyanska et al. and ascribed to a low density of nonradiative recombination centers. ${ }^{46}$

The temperature dependence of the PL intensity obtained for $4.5 \mathrm{~nm} \mathrm{PbS}$ QDs is shown in Figure 7 by blue circles and demonstrates a very different behavior. Besides the decrease of PL intensity with decreasing temperature, we observed an unexpected rise in the PL intensity above $150 \mathrm{~K}$. This indicates that at higher temperatures an additional mechanism of radiative recombination is contributing to the PL. It has been shown that $\mathrm{PbS} \mathrm{QDs}$ possess a complicated low-energy electronic structure, and radiative recombination drastically depends on QD size and temperature. ${ }^{22,47}$ Several research groups have reported splitting of the lowest electronic state into "dark" and "bright" states, as shown schematically in Figure 8, assuming the "dark" state can either be highly emissive, ${ }^{22,31,32}$ slightly emissive, ${ }^{34}$ or nonemissive. ${ }^{48}$

Since the PL band observed in our experiments is highly symmetric for both 3.7 and $4.5 \mathrm{~nm}$ QDs in the NSM, we consider a contribution from only one radiative transition from a "bright" state to the total PL signal. However, when the energy gap, $\Delta E$, between "dark" and "bright" states is small enough, thermally activated reverse transitions with a rate constant $k_{\mathrm{BD}}$ increase the population of the "bright" state, leading to a growth in the PL signal. This mechanism is illustrated by the red arrow in Figure 8. It is known that energy splitting increases strongly with decreasing QD size; ${ }^{22,32}$ thus, this mechanism may be negligible for $3.7 \mathrm{~nm}$ QDs.

Taking into account the radiative and nonradiative transitions with the rate constants $k_{\mathrm{r}}$ and $k_{\mathrm{nr}}$ and internal conversion to the "dark" state with the rate $k_{\mathrm{DB}}$, the population of the "bright" state $N_{\mathrm{B}}$ will be described by the following equation:

$$
\frac{\mathrm{d} N_{\mathrm{B}}}{\mathrm{d} t}=-\left(k_{\mathrm{r}}+k_{\mathrm{nr}}+k_{\mathrm{DB}}\right) N_{\mathrm{B}}+k_{\mathrm{BD}} N_{\mathrm{D}}
$$




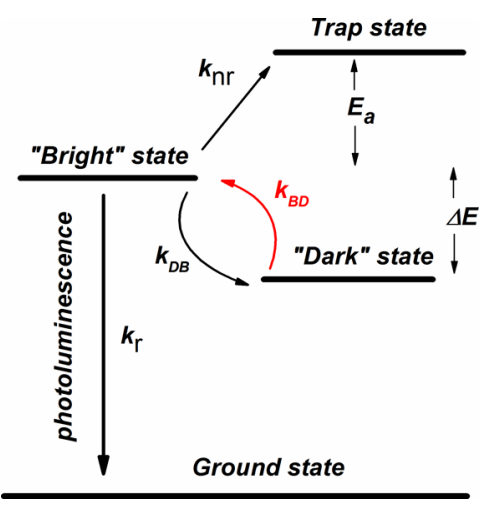

Figure 8. Sketch of the exciton relaxation for the $\mathrm{PbS}$ QDs studied. Depopulation of the "bright" state occurs via radiative recombination to the ground state with rate $k_{\mathrm{r}}$, nonradiative recombination to the trap state separated by activation energy $E_{\mathrm{a}}$, with rate $k_{\mathrm{nr}}$, and to the "dark" state with rate $k_{\mathrm{DB}}$. Simultaneously, the population of the "bright" state can be increased via thermally activated transitions from the "dark" state at a rate $k_{\mathrm{BD}}$, which depends on the energy gap $\Delta E$ between the "dark" and "bright" states.

$$
k_{\mathrm{BD}} \sim k_{\mathrm{DB}} \exp \left(-\frac{\Delta E}{k_{\mathrm{B}} T}\right)
$$

Equation 3 can be modified to describe the temperature dependence of the PL intensity for $4.5 \mathrm{~nm}$ QDs:

$$
I_{\mathrm{PL}}=\frac{I_{0}+N_{\mathrm{D}} \exp \left(-\frac{\Delta E}{k_{\mathrm{B}} T}\right)}{1+A \exp \left(-\frac{E_{\mathrm{A}}}{k_{\mathrm{B}} T}\right)}
$$

where $N_{\mathrm{D}}$ is a temperature-independent parameter characterizing the population of the "dark" state. ${ }^{36}$ Equation 6 is a good fit to the experimental data, as shown in Figure 6, and gives an energy gap, $\Delta E$, between the "dark" and "bright" states, of 52 $\mathrm{meV}$ for $4.5 \mathrm{~nm}$ QDs, in agreement with data from the literature. $^{22,47,49}$ The estimated activation energy $E_{\mathrm{A}}$ for $4.5 \mathrm{~nm}$ QDs equals $34 \mathrm{meV}$.

An additional feature in the PL temperature dependence of $4.5 \mathrm{~nm}$ QDs has been observed at $250 \mathrm{~K}$ (Figure 7). Since this temperature coincides with the melting point of $\mathrm{CCl}_{4}$, we speculate that it may be related to the presence of residual solvent within the pores. In our case, upon solvent melting at $250 \mathrm{~K}$, the vibrational modes of liquid $\mathrm{CCl}_{4}$ become active and further increases in the QD PL intensity may be inhibited by some competing process, such as electronic-to-vibrational energy transfer. ${ }^{33}$

It worth noting that other mechanisms could also lead to changes in the temperature dependence of PL intensity; e.g., the influence of oxygen exposure ${ }^{50}$ and FRET $^{51,52}$ have also been considered for PbS QDs. However, in our case, the first factor can be excluded since both 3.7 and $4.5 \mathrm{~nm}$ QDs would experience similar effects due to oxygen. In addition, FRET is negligible at the low QD concentrations used.

Close-Packed Regime. Besides the opportunity to study optical properties of isolated QDs, the NSM allows the investigation of various interactions at the nanoscale. The NSM is suitable for the experimental study of interactions between semiconductor QDs, plasmonic metal nanoparticles, and biological objects. One example is the Förster resonance energy transfer (FRET) between donor and acceptor nanoparticles.

FRET between two QDs of different sizes exhibits wellknown features, allowing an evaluation of the average interdot distance. Since we did not observe any spectral features suggesting QD-QD interaction for the samples prepared from the colloidal solutions with QD concentrations of $10^{-5} \mathrm{M}$, the QD density in the NSM must be increased. To achieve a higher density in the QD distribution in the NSM matrix, we increased the concentration of the initial QD solution to $\sim 10^{-4} \mathrm{M}$. We prepared the samples with $3.7 \mathrm{~nm}$ QDs, $4.5 \mathrm{~nm}$ QDs, and a mixture of 3.7 and $4.5 \mathrm{~nm}$ QDs at a $1: 1$ concentration ratio, keeping the total $\mathrm{QD}$ concentration at $\sim 10^{-4} \mathrm{M}$ for all $\mathrm{QD}$ solutions. PL spectra for $3.7 \mathrm{~nm}$ QDs, $4.5 \mathrm{~nm}$ QDs, and their mixture in the NSM are shown in Figure 9a. The spectrum of the QD mixture indicates FRET is taking place between QDs of different sizes, resulting in both quenching of donor PL ( $3.7 \mathrm{~nm}$ QDs) and enhancement of acceptor PL (4.5 nm QDs). Additional evidence for this comes from transient PL analysis. PL decay curves have been recorded at $1000 \mathrm{~nm}$ for the samples with a donor-QD and QD mixture and are shown in Figure 9b. At this wavelength, PL from the QD mixture arises from the donor-QDs only and may indicate a quenching process. Indeed, the decay curve for the donor-QDs in the mixture is characterized by a smaller amplitude and shorter PL
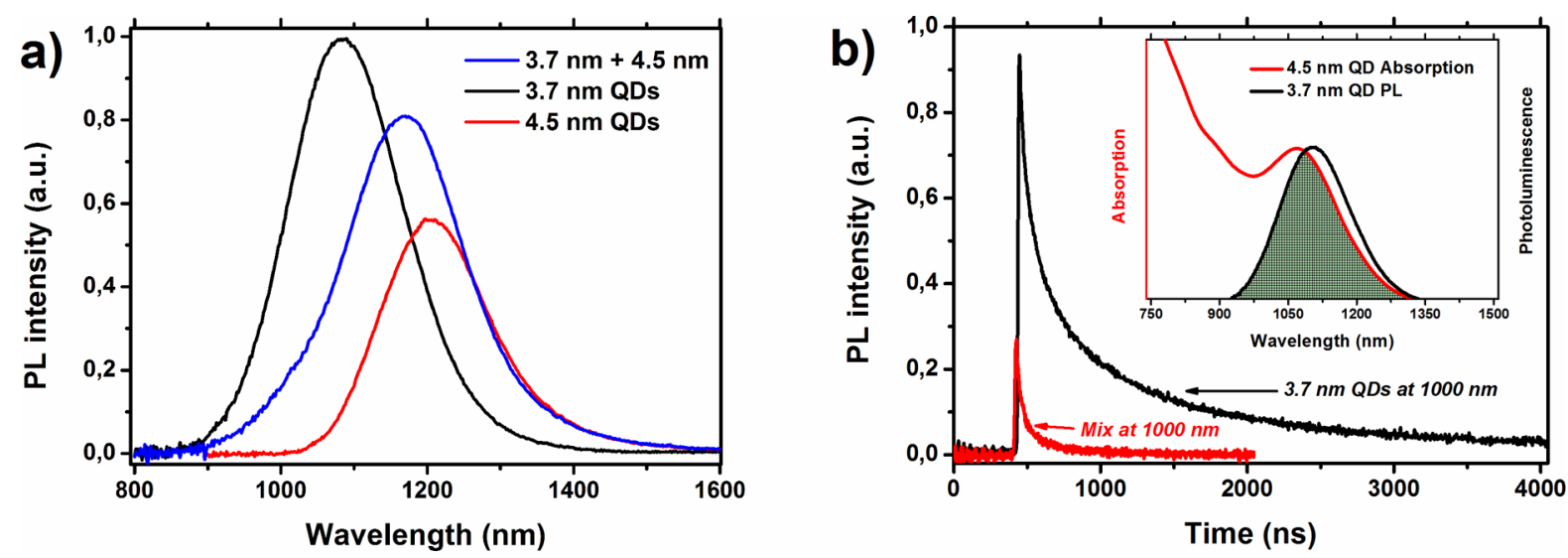

Figure 9. (a) PL spectra for $3.7 \mathrm{~nm}$ QDs (black), $4.5 \mathrm{~nm}$ QDs (red), and their mixture in NSM (blue). (b) PL decay curves recorded at $1000 \mathrm{~nm}$ for the samples with the donor- $\mathrm{QD}$ and $\mathrm{QD}$ mixture. The inset demonstrates the overlap between the donor PL spectrum and the acceptor absorption spectrum. 
lifetime in comparison to the reference sample with $3.7 \mathrm{~nm}$ QDs only. This confirms FRET is occurring from the smaller QDs to the larger ones, indicating a short interparticle distance. A FRET efficiency of $74 \%$ was extracted from the donor PL lifetimes using the expression ${ }^{53}$

$$
E=1-\frac{\tau_{\mathrm{DA}}}{\tau_{\mathrm{D}}}
$$

where $\tau_{\mathrm{DA}}$ and $\tau_{\mathrm{D}}$ are the PL lifetimes of the donor QD in the presence and absence of a QD acceptor, respectively. The Forster radii, which characterize the distance at which energy transfer efficiency equals $50 \%$, can be estimated as follows: ${ }^{53}$

$$
R_{\mathrm{F}}{ }^{6}=\frac{9000 \ln 10 k^{2} Q_{\mathrm{D}}}{128 \pi N_{\mathrm{A}} n^{4}} \int_{0}^{\infty} F_{\mathrm{D}}(\lambda) \varepsilon_{\mathrm{A}}(\lambda) \lambda^{4} \mathrm{~d} \lambda
$$

where $k^{2}$ equals $2 / 3$ and is the orientational factor, the QDdonor quantum yield $Q_{D}$ is estimated to be $\sim 8 \%$ in the NSM, the refractive index $n$ of the NSM with a porosity of $52 \%$ with embedded QDs is estimated to be 1.222, and the overlap integral describes the spectral overlap between donor emission and acceptor extinction. $R_{\mathrm{F}}$ equals $8.7 \mathrm{~nm}$, and the corresponding average interdot distance, which can be estimated using the FRET efficiency, equals $7.3 \mathrm{~nm}$. Summation of the calculated interdot distance, oleic acid length, and QDs radii gives a distance close to the diameter of the pores in the NSM, indicating the interaction of two QDs of different sizes in a pore. That correlates well with the equimolar concentration ratio for the sample studied.

\section{CONCLUSIONS}

We studied the optical properties of lead sulfide QDs of two different sizes in a nanoporous silicate glass matrix. We showed that the use of a nanoporous glass matrix allowed us to obtain samples with reproducible optical characteristics, both for isolated noninteracting QDs and for QDs forming close-packed structures. Low-temperature PL analysis performed for QDs with sizes of 3.7 and $4.5 \mathrm{~nm}$ showed that the coefficient of the temperature shift of PL position changes sign with reducing QD size. This supports a model of size-dependent contributions from thermal expansion, mechanical strain, and electronphonon coupling to the temperature-induced shift of the lowest energy absorption peak of PbS QDs. Different behaviors of the PL intensity with temperature for QDs of different size was observed and explained by size-dependent splitting of the lowest electronic state and a variation of the energy gap between "dark" and "bright" electronic states.

\section{AUTHOR INFORMATION}

\section{Corresponding Author}

*(A.P.L.) E-mail: litvin88@gmail.com.

\section{ORCID}

Aleksandr P. Litvin: 0000-0001-5261-3210

\section{Notes}

The authors declare no competing financial interest.

\section{ACKNOWLEDGMENTS}

The authors thank the Ministry of Education and Science of the Russian Federation (State task no. 16.8981.2017/BCh and Grant 14.B25.31.0002) for financial support. A.P.L. thanks the Ministry of Education of the Russian Federation for financial support (scholarship of the President of the Russian Federation for young scientists and graduate students, СП-1841.2015.1)

\section{REFERENCES}

(1) Kovalenko, M. V.; Manna, L.; Cabot, A.; Hens, Z.; Talapin, D. V.; Kagan, C. R.; Klimov, V. I.; Rogach, A. L.; Reiss, P.; Milliron, D. J.; et al. Prospects of Nanoscience with Nanocrystals. ACS Nano 2015, 9 (2), $1012-1057$.

(2) Carey, G. H.; Abdelhady, A. L.; Ning, Z.; Thon, S. M.; Bakr, O. M.; Sargent, E. H. Colloidal Quantum Dot Solar Cells. Chem. Rev. 2015, 115 (23), 12732-12763.

(3) Duan, J.; Zhang, H.; Tang, Q.; He, B.; Yu, L. Recent Advances in Critical Materials for Quantum Dot-Sensitized Solar Cells: A Review. J. Mater. Chem. A 2015, 3 (34), 17497-17510.

(4) Ning, Z.; Wang, R.; Shang, Y.; Kanjanaboos, P.; Zhou, W.; Sargent, E. H. Colloidal Quantum Dot Ligand Engineering for High Performance Solar Cells. Energy Environ. Sci. 2016, 9 (4), 1130-1143.

(5) Saran, R.; Curry, R. J. Lead Sulphide Nanocrystal Photodetector Technologies. Nat. Photonics 2016, 10 (2), 81-92.

(6) Hu, C.; Gassenq, A.; Justo, Y.; Devloo-Casier, K.; Chen, H.; Detavernier, C.; Hens, Z.; Roelkens, G. Air-Stable Short-Wave Infrared $\mathrm{PbS}$ Colloidal Quantum Dot Photoconductors Passivated with Al2O3 Atomic Layer Deposition. Appl. Phys. Lett. 2014, 105 (17), 171110.

(7) Konstantatos, G.; Badioli, M.; Gaudreau, L.; Osmond, J.; Bernechea, M.; Garcia de Arquer, F. P.; Gatti, F.; Koppens, F. H. L. Hybrid Graphene-Quantum Dot Phototransistors with Ultrahigh Gain. Nat. Nanotechnol. 2012, 7 (6), 363-368.

(8) Lee, J. W.; Kim, D. Y.; So, F. Unraveling the Gain Mechanism in High Performance Solution-Processed PbS Infrared PIN Photodiodes. Adv. Funct. Mater. 2015, 25 (8), 1233-1238.

(9) Huang, Y. Q.; Zhu, R. J.; Kang, N.; Du, J.; Xu, H. Q. Photoelectrical Response of Hybrid Graphene-PbS Quantum Dot Devices. Appl. Phys. Lett. 2013, 103 (14), 143119.

(10) Bourdakos, K. N.; Dissanayake, D. M. N. M.; Lutz, T.; Silva, S. R. P.; Curry, R. J. Highly Efficient near-Infrared Hybrid OrganicInorganic Nanocrystal Electroluminescence Device. Appl. Phys. Lett. 2008, 92 (15), 153311.

(11) Gong, X.; Yang, Z.; Walters, G.; Comin, R.; Ning, Z.; Beauregard, E.; Adinolfi, V.; Voznyy, O.; Sargent, E. H. Highly Efficient Quantum Dot near-Infrared Light-Emitting Diodes. Nat. Photonics 2016, 10 (4), 253-257.

(12) van Veggel, F. C. J. M. Near-Infrared Quantum Dots and Their Delicate Synthesis, Challenging Characterization, and Exciting Potential Applications. Chem. Mater. 2014, 26 (1), 111-122.

(13) He, J.; Wang, Y.; Li, R.; Yuan, X.; Xu, S.; Zhang, L. Tunable and White Light Emitting AlPO ${ }_{4}$ Mesoporous Glass by Design of Inorganic/organic Luminescent Species. APL Mater. 2015, 3 (4), 046101.

(14) Song, H.; Lee, S. Photoluminescent (CdSe)ZnS Quantum Dotpolymethylmethacrylate Polymer Composite Thin Films in the Visible Spectral Range. Nanotechnology 2007, 18 (5), 055402.

(15) Reda, S. M. Synthesis and Optical Properties of CdS Quantum Dots Embedded in Silica Matrix Thin Films and Their Applications as Luminescent Solar Concentrators. Acta Mater. 2008, 56 (2), 259-264.

(16) Schüler, A.; Python, M.; del Olmo, M. V.; de Chambrier, E. Quantum Dot Containing Nanocomposite Thin Films for Photoluminescent Solar Concentrators. Sol. Energy 2007, 81 (9), 11591165.

(17) Jeong, S.; Lee, J.; Nam, J.; Im, K.; Hur, J.; Park, J.-J.; Kim, J.-M.; Chon, B.; Joo, T.; Kim, S. One-Step Preparation of Strongly Luminescent and Highly Loaded CdSe Quantum Dot-Silica Films. J. Phys. Chem. C 2010, 114 (34), 14362-14367.

(18) Solomeshch, O.; Tessler, N. Research Update: Preserving the Photoluminescence Efficiency of near Infrared Emitting Nanocrystals When Embedded in a Polymer Matrix. APL Mater. 2016, 4 (4), 040702.

(19) Andreeva, O. V.; Belousova, I. M.; Veselova, T. V.; Gavronskaya, E. A.; Grigorev, V. A.; Obyknovennay, I. E.; Skobelev, A. G.; Cherkasov, A. S. The Possibility of Using Fullerene-Saturated Porous 
Glasses for the Optical Limitation of Laser Radiation. J. Opt. Technol. 2001, 68 (12), 882.

(20) Andreeva, O. V.; Sidorov, A. I.; Stasel'ko, D. I.; Khrushcheva, T. A. Synthesis and Optical Properties of Hybrid "plasmon-Exciton" Nanostructures Based on Ag-AgI in Nanoporous Silica Glass. Phys. Solid State 2012, 54 (6), 1293-1297.

(21) Orlova, A. O.; Gromova, Y. A.; Maslov, V. G.; Andreeva, O. V.; Baranov, A. V.; Fedorov, A. V.; Prudnikau, A. V.; Artemyev, M. V.; Berwick, K. Reversible Photoluminescence Quenching of CdSe/ZnS Quantum Dots Embedded in Porous Glass by Ammonia Vapor. Nanotechnology 2013, 24, 335701.

(22) Ushakova, E. V.; Litvin, A. P.; Parfenov, P. S.; Fedorov, A. V.; Artemyev, M. V.; Prudnikau, A. V.; Rukhlenko, I. D.; Baranov, A. V. Anomalous Size-Dependent Decay of Low-Energy Luminescence from $\mathrm{PbS}$ Quantum Dots in Colloidal Solution. ACS Nano 2012, 6 (10), 8913-8921.

(23) Litvin, A. P.; Parfenov, P. S.; Ushakova, E. V.; Fedorov, A. V.; Artemyev, M. V.; Prudnikau, A. V.; Golubkov, V. V.; Baranov, A. V. $\mathrm{PbS}$ Quantum Dots in a Porous Matrix: Optical Characterization. J. Phys. Chem. C 2013, 117 (23), 12318-12324.

(24) Litvin, A. P.; Ushakova, E. V.; Parfenov, P. S.; Fedorov, A. V.; Baranov, A. V. FRET between Close-Packed Quasi-Monodispersed PbS QDs in a Porous Matrix. J. Phys. Chem. C 2014, 118 (12), 65316535.

(25) Parfenov, P. S.; Litvin, A. P.; Ushakova, E. V.; Fedorov, A. V.; Baranov, A. V.; Berwick, K. Note: Near Infrared Spectral and Transient Measurements of PbS Quantum Dots Luminescence. Rev. Sci. Instrum. 2013, 84 (11), 116104.

(26) Parfenov, P. S.; Litvin, A. P.; Baranov, A. V.; Veniaminov, A. V.; Ushakova, E. V. Calibration of the Spectral Sensitivity of Instruments for the near Infrared Region. J. Appl. Spectrosc. 2011, 78 (3), 433-439.

(27) Kagan, C.; Murray, C.; Bawendi, M. Long-Range Resonance Transfer of Electronic Excitations in Close-Packed CdSe QuantumDot Solids. Phys. Rev. B: Condens. Matter Mater. Phys. 1996, 54 (12), 8633-8643.

(28) Ren, F.; Lindley, S. A.; Zhao, H.; Tan, L.; Gonfa, B. A.; Pu, Y.C.; Yang, F.; Liu, X.; Vidal, F.; Zhang, J. Z.; et al. Towards Understanding Unusual Photoluminescence Intensity Variation of Ultrasmall Colloidal PbS Quantum Dots with the Formation of Thin CdS Shell. Phys. Chem. Chem. Phys. 2016, 18 (46), 31828.

(29) Clark, S. W.; Harbold, J. M.; Wise, F. W. Resonant Energy Transfer in PbS Quantum Dots. J. Phys. Chem. C 2007, 111 (20), $7302-7305$

(30) Warner, J. H.; Thomsen, E.; Watt, A. R.; Heckenberg, N. R.; Rubinsztein-Dunlop, H. Time-Resolved Photoluminescence Spectroscopy of Ligand-Capped PbS Nanocrystals. Nanotechnology 2005, 16 (2), 175-179.

(31) Fernée, M. J.; Thomsen, E.; Jensen, P.; Rubinsztein-Dunlop, H. Highly Efficient Luminescence from a Hybrid State Found in Strongly Quantum Confined PbS Nanocrystals. Nanotechnology 2006, 17 (4), 956-962.

(32) Caram, J. R.; Bertram, S. N.; Utzat, H.; Hess, W. R.; Carr, J. A.; Bischof, T. S.; Beyler, A. P.; Wilson, M. W. B.; Bawendi, M. G. PbS Nanocrystal Emission Is Governed by Multiple Emissive States. Nano Lett. 2016, 16 (10), 6070-6077.

(33) Aharoni, A.; Oron, D.; Banin, U.; Rabani, E.; Jortner, J. LongRange Electronic-to-Vibrational Energy Transfer from Nanocrystals to Their Surrounding Matrix Environment. Phys. Rev. Lett. 2008, 100 (5), 57404 .

(34) Gaponenko, M. S.; Lutich, A. A.; Tolstik, N. A.; Onushchenko, A. A.; Malyarevich, A. M.; Petrov, E. P.; Yumashev, K. V. TemperatureDependent Photoluminescence of $\mathrm{PbS}$ Quantum Dots in Glass: Evidence of Exciton State Splitting and Carrier Trapping. Phys. Rev. B: Condens. Matter Mater. Phys. 2010, 82 (12), 125320.

(35) Morello, G.; De Giorgi, M.; Kudera, S.; Manna, L.; Cingolani, R.; Anni, M. Temperature and Size Dependence of Nonradiative Relaxation and Exciton-Phonon Coupling in Colloidal CdTe Quantum Dots. J. Phys. Chem. C 2007, 111 (16), 5846-5849.
(36) Jing, P.; Zheng, J.; Ikezawa, M.; Liu, X.; Lv, S.; Kong, X.; Zhao, J.; Masumoto, Y. Temperature-Dependent Photoluminescence of CdSe-Core CdS/CdZnS/ZnS-Multishell Quantum Dots. J. Phys. Chem. C 2009, 113 (31), 13545-13550.

(37) Madelung, O.; Rössler, U.; Schulz, M. Lead Sulfide (PbS) Phonon Dispersion and Frequencies. In Landolt-Börnstein - Group III Condensed Matter 41C (Non-Tetrahedrally Bonded Elements and Binary Compounds I); Springer-Verlag: Berlin, pp 1-3.

(38) Olkhovets, A.; Hsu, R.-C.; Lipovskii, A.; Wise, F. W. SizeDependent Temperature Variation of the Energy Gap in Lead-Salt Quantum Dots. Phys. Rev. Lett. 1998, 81 (16), 3539-3542.

(39) Dey, P.; Paul, J.; Bylsma, J.; Karaiskaj, D.; Luther, J. M.; Beard, M. C.; Romero, a. H. Origin of the Temperature Dependence of the Band Gap of PbS and PbSe Quantum Dots. Solid State Commun. 2013, 165, 49-54.

(40) Zhao, H.; Liang, H.; Vidal, F.; Rosei, F.; Vomiero, A.; Ma, D. Size Dependence of Temperature-Related Optical Properties of $\mathrm{PbS}$ and $\mathrm{PbS} / \mathrm{CdS}$ Core/Shell Quantum Dots. J. Phys. Chem. C 2014, 118 (35), 20585-20593.

(41) Krustok, J.; Collan, H.; Hjelt, K. Does the Low-Temperature Arrhenius Plot of the Photoluminescence Intensity in CdTe Point towards an Erroneous Activation Energy? J. Appl. Phys. 1997, 81 (3), $1442-1445$

(42) Stadler, W.; Hofmann, D. M.; Alt, H. C.; Muschik, T.; Meyer, B. K.; Weigel, E.; Müller-Vogt, G.; Salk, M.; Rupp, E.; Benz, K. W. Optical Investigations of Defects in Cd1-xZnxTe. Phys. Rev. B: Condens. Matter Mater. Phys. 1995, 51 (16), 10619-10630.

(43) Krustok, J.; Valdna, V.; Hjelt, K.; Collan, H. Deep Center Luminescence in P-Type CdTe. J. Appl. Phys. 1996, 80 (3), 1757.

(44) Schwarzl, T.; Kaufmann, E.; Springholz, G.; Koike, K.; Hotei, T.; Yano, M.; Heiss, W. Temperature-Dependent Midinfrared Photoluminescence of Epitaxial $\mathrm{PbTe} / \mathrm{CdTe}$ Quantum Dots and Calculation of the Corresponding Transition Energy. Phys. Rev. B: Condens. Matter Mater. Phys. 2008, 78 (16), 165320.

(45) Abel, K. A.; Qiao, H.; Young, J. F.; van Veggel, F. C. J. M. FourFold Enhancement of the Activation Energy for Nonradiative Decay of Excitons in $\mathrm{PbSe} / \mathrm{CdSe}$ Core/Shell versus PbSe Colloidal Quantum Dots. J. Phys. Chem. Lett. 2010, 1 (15), 2334-2338.

(46) Turyanska, L.; Patané, A.; Henini, M.; Hennequin, B.; Thomas, N. R. Temperature Dependence of the Photoluminescence Emission from Thiol-Capped PbS Quantum Dots. Appl. Phys. Lett. 2007, 90 (10), 101913

(47) Litvin, A. P.; Parfenov, P. S.; Ushakova, E. V.; Simões Gamboa, A. L.; Fedorov, A. V.; Baranov, A. V. Size and Temperature Dependencies of the Low-Energy Electronic Structure of $\mathrm{PbS}$ Quantum Dots. J. Phys. Chem. C 2014, 118 (35), 20721-20726.

(48) Yue, F.; Tomm, J. W.; Kruschke, D. Spontaneous and Stimulated Emission Dynamics of PbS Quantum Dots in a Glass Matrix. Phys. Rev. B: Condens. Matter Mater. Phys. 2013, 87 (19), 195314.

(49) Litvin, A. P.; Parfenov, P. S.; Ushakova, E. V.; Fedorov, A. V.; Artemyev, M. V.; Prudnikau, A. V.; Cherevkov, S. A.; Rukhlenko, I. D.; Baranov, A. V. Size-Dependent Room-Temperature Luminescence Decay from PbS Quantum Dots. Proc. SPIE 2012, 8564, 85641Z.

(50) Chappell, H. E.; Hughes, B. K.; Beard, M. C.; Nozik, A. J.; Johnson, J. C. Emission Quenching in PbSe Quantum Dot Arrays by Short-Term Air Exposure. J. Phys. Chem. Lett. 2011, 2 (8), 889-893.

(51) Lü, W.; Kamiya, I.; Ichida, M.; Ando, H. Temperature Dependence of Electronic Energy Transfer in PbS Quantum Dot Films. Appl. Phys. Lett. 2009, 95 (8), 083102.

(52) Rinnerbauer, V.; Egelhaaf, H.-J.; Hingerl, K.; Zimmer, P.; Werner, S.; Warming, T.; Hoffmann, A.; Kovalenko, M.; Heiss, W.; Hesser, G.; et al. Energy Transfer in Close-Packed PbS Nanocrystal Films. Phys. Rev. B: Condens. Matter Mater. Phys. 2008, 77 (8), 85322.

(53) Lakowicz, J. R. Principles of Fluorescence Spectroscopy; Springer Science \& Business Media: 2013. 